\title{
Rational iterated function system for positive/monotonic shape preservation
}

\author{
AKB Chand ${ }^{1 *}$, N Vijender ${ }^{1}$ and RP Agarwal ${ }^{2,3}$
}

\footnotetext{
*Correspondence: chand@iitm.ac.in

${ }^{1}$ Department of Mathematics,

Indian Institute of Technology

Madras, Chennai, 600036, India

Full list of author information is

available at the end of the article
}

\begin{abstract}
In this paper we consider the (inverse) problem of determining the iterated function system (IFS) which produces a shaped fractal interpolant. We develop a new type of rational IFS by using functions of the form $\frac{E_{i}}{F_{i}}$, where $E_{i}$ are cubics and $F_{i}$ are preassigned quadratics having 3-shape parameters. The fixed point of the developed rational cubic IFS is in $\mathcal{C}^{1}$, but its derivative varies from a piecewise differentiable function to a continuous nowhere differentiable function. An upper bound of the uniform error between the fixed point of a rational IFS and an original function $\Phi \in \mathcal{C}^{4}$ is deduced for the convergence results. The automatic generations of the scaling factors and shape parameters in the rational IFS are formulated so that its fixed point preserves the positive/monotonic features of prescribed data. The presence of scaling factors provides additional freedom to the shape of the fractal interpolant over its classical counterpart in the modeling of discrete data.
\end{abstract}

\section{Introduction}

Setting a novel platform for the approximation of natural objects such as trees, clouds, feathers, leaves, flowers, landscapes, glaciers, galaxies, and torrents of water, Mandelbrot [1] introduced the term fractal in the literature. Since fractals capture the non-linear structures of various objects effectively, the fractal geometry has been successfully used in different problems in applied sciences and engineering [1-4]. The iterated function system (IFS) was introduced by Hutchinson [5] for the construction of various types of fractal sets, and popularized by Barnsley [6]. An IFS is a dynamical system consisting of a finite collection of continuous maps. Based on the IFS theory, Barnsley [7] constructed a class of functions that are known as FIFs. The graph of a FIF is the fixed point of an IFS. Also a FIF is the fixed point of the Read-Bajraktarević operator on a suitable function space. Common features between a FIF and a piecewise polynomial interpolation are that they are geometrical in nature, and they can be computed rapidly, but the main difference is the fractal character, i.e., a FIF satisfies a functional relation related to the self-similarity on smaller scales. In the direction of smooth fractal curves, Barnsley and Harington [8] initiated the construction of a restricted class of differentiable FIF or $\mathcal{C}^{k}$-FIF that interpolates the prescribed data if the values of $\Phi^{(p)}, p=1,2, \ldots, k$, at the initial end point of the interval are given, where $\Phi$ is the original function. This method is based on the recursive nature of an algorithm, and specifying the boundary conditions similar to the classical splines was found to be quite difficult to handle in this construction. The fractal splines with general boundary conditions have been studied recently [9-13] by restricting their IFSs parameters suitably.

@2014 Chand et al.; licensee Springer. This is an Open Access article distributed under the terms of the Creative Commons Attribution License (http://creativecommons.org/licenses/by/2.0), which permits unrestricted use, distribution, and reproduction in any medium, provided the original work is properly cited. 
The motivation of this work is the research on different types of splines by several authors; see, for instance, Schmidt and Heß [14], Fritsch and Carlson [15], Schumaker [16], and Brodlie and Butt [17], and references therein. The uniqueness of spline representation for a given data set turns out to be a disadvantage for shape modification problems. The use of rational functions with the shape parameters was introduced by Späth [18] to preserve different geometric properties attached to a given set of data. Rational interpolants are often used in data visualization problems due to their excellent asymptotic properties, capability to model complicated smooth structures, better interpolation properties, and excellent extrapolating powers. Gregory and Delbourgo [19] introduced the rational cubic spline with one family of shape parameters, and this work inspired a large amount of research in shape-preserving rational spline interpolations, see $[20,21]$ and references therein.

In this paper, we introduce the rational cubic IFS with 3-shape parameters in each subinterval of the interpolation domain such that its fixed point generalizes the corresponding classical rational cubic spline functions [20]. The developed rational cubic spline FIF is bounded, and is unique by fixed point theory for a given set of scaling factors and shape parameters. Because of the recursive nature of FIF, the necessary conditions for monotonicity on the derivative values at knots alone may not ensure the monotonicity of a rational cubic fractal interpolant for a given monotonic data. Based on the appropriate condition on the rational IFS parameters: (i) the scaling factors that depend only on given data, and (ii) the shape parameters that depend on both the interpolation data and scaling factors, we construct the shape-preserving rational cubic FIFs for a prescribed positive and/or monotonic data. By varying the scaling factors (within the shape-preserving interval) and shape parameters (according to the conditions derived in our theory), we can make the fixed point of a rational cubic IFS more pleasant and suitable for aesthetic requirements in a modeling problem. The proposed method is suitable for the shape-preserving interpolation problems where a data set originates from an unknown function $\Phi \in \mathcal{C}^{1}$ and its derivative $\Phi^{\prime}$ is a continuous nowhere differentiable function, for instance, the motion of single inverted pendulum in non-linear control theory [22].

Comparison of the proposed rational cubic FIF over some existing schemes:

- When all the scaling factors are zero, the proposed rational cubic FIF reduces to the classical rational cubic interpolant [20], see Remark 1, Section 3.

- To generate shape-preserving interpolants, our construction does not need additional knots in contrast to methods due to Schumaker [16] and Brodlie and Butt [17], which require additional knots for the shape-preserving interpolants.

- The classical interpolants $[15,23]$ are suitable only for monotonicity interpolation whereas the proposed rational cubic FIF is suitable for both monotonicity and positivity interpolation. Moreover, the rational quadratic interpolant [23] is a special case of our rational cubic FIF for the particular choice of the scaling factors and shape parameters, see Remark 2, Section 3.

- For given monotonic data, the monotonic curve generated by the rational quadratic interpolant [23] is unique for fixed shape parameters, whereas for the same monotonic data an infinite number of monotonic curves will be obtained using our rational FIF by suitable modifications in the associated scaling factors. Thus, when the shape parameters are incapable to change the shape of an interpolant in given intervals, then the scaling factors can be used to alter the shape of the interpolant in our method. 
- Where monotonicity is concerned, our construction does not need an additional condition on derivatives at knots except for the necessary conditions. But the construction of Fritsch and Carlson [15] needs some restrictions on derivatives at knots apart from the necessary conditions for the same problem.

- The derivatives of the shape-preserving interpolants [15-17,23] are piecewise smooth, whereas the derivative of our rational cubic FIF may be piecewise smooth to a non-differentiable function according to the choice of the scaling factors. Owing to this special feature, the proposed method is preferable over the classical shape-preserving interpolants when the approximation is taken for data originating with an unknown function $\Phi \in \mathcal{C}^{1}$ having a shape with fractality in $\Phi^{\prime}$.

This paper is organized as follows. In Section 2, the general constructions of fractal interpolants and $\mathcal{C}^{r}$-rational cubic FIFs based on IFSs are summarized. Section 3 is devoted to the construction of a suitable rational IFS so that its fixed point is the desired interpolant that can be used for shape preservation. Then we deduce an upper bound of the uniform error bound between the original function and the rational cubic FIF. The fixed point of this rational IFS does not follow any shape constraints. The restrictions on the rational IFS parameters are deduced for a positivity shape in Section 4, and the results are illustrated with suitably chosen examples. In Section 5 , the monotonicity problem is considered through the developed rational cubic IFS.

\section{IFS for fractal functions}

Let $x_{1}<\cdots<x_{n}$ be a partition of $I=\left[x_{1}, x_{n}\right]$. Let $f_{i}$ be the value of original function at $x_{i}$, $i=1,2, \ldots, n$. Denote $I_{i}=\left[x_{i}, x_{i+1}\right], C=I \times D, C_{i}=I_{i} \times D$, and let $D$ be a compact sub-set of $\mathbb{R}$ such that $f_{i} \in D, i=1,2, \ldots, n$. Let $L_{i}(x)=a_{i} x+b_{i}: I \rightarrow I_{i}, i=1,2, \ldots, n-1$, be the contractive homeomorphisms such that

$$
L_{i}\left(x_{1}\right)=x_{i}, \quad L_{i}\left(x_{n}\right)=x_{i+1} .
$$

It is easy to verify that $\left\{I ; L_{i}(x), i=1,2, \ldots, n-1\right\}$ is a just touching hyperbolic IFS whose unique fixed point is

$$
I=\bigcup_{i=1}^{n-1} L_{i}(I) .
$$

Let $F_{i}(x, f)=\xi_{i} f+q_{i}(x),\left|\xi_{i}\right|<1, i=1,2, \ldots, n-1$, be the continuous real-valued functions on $C$ such that

$$
F_{i}\left(x_{1}, f_{1}\right)=f_{i}, \quad F_{i}\left(x_{n}, f_{n}\right)=f_{i+1},
$$

and $q_{i}: I \rightarrow \mathbb{R}, i=1,2, \ldots, n-1$, are the suitable continuous functions. Now define the functions $w_{i}: C \rightarrow C_{i}, \forall i=1,2, \ldots, n-1$, as $w_{i}(x, f)=\left(L_{i}(x), F_{i}(x, f)\right)$ for every $(x, f) \in C$. Then $\mathcal{I} \equiv\left\{C ; w_{i}(x, f), i=1,2, \ldots, n-1\right\}$ is called an IFS related to a given interpolation data $\left\{\left(x_{i}, f_{i}\right), i=1,2, \ldots, n\right\}$. According to [7], the IFS $\mathcal{I}$ has a unique fixed point $G$ which is the graph of a continuous function $\phi: I \rightarrow \mathbb{R}, \phi\left(x_{i}\right)=f_{i}, i=1,2, \ldots, n$. The function $\phi$ is called a FIF generated by the IFS $\mathcal{I}$, and it takes the form

$$
\phi\left(L_{i}(x)\right)=\xi_{i} \phi(x)+q_{i}(x), \quad x \in\left[x_{1}, x_{n}\right]
$$


The existence of a spline FIF based on a polynomial IFS is given in [8]. We have extended this result to the rational IFS with 3 -shape parameters in the following.

Theorem 1 Let $\left\{\left(x_{i}, f_{i}\right), i=1,2, \ldots, n\right\}$ be a given data set, where $d_{i}$ are the slope at $x_{i}$, and $d_{i}^{(k)}(k=2, \ldots, r)$ are the $k$ th derivative values at $x_{i}$ for $i=1,2, \ldots, n$. Consider the rational IFS $\mathcal{I}^{*} \equiv\left\{I \times D_{1} ; w_{i}(x, f)=\left(L_{i}(x), F_{i}(x, f)\right), i=1,2, \ldots, n-1\right\}$, where $L_{i}(x)=a_{i} x+b_{i}$ satisfies equation (1), $D_{1}$ is a suitable compact sub-set of $\mathbb{R} . F_{i}(x, f)=a_{i}^{r}\left(\xi_{i} f+q_{i}(x)\right), \frac{\Omega_{i, 1}(x)}{\Omega_{i, 2}(x)}, \Omega_{i, 1}(x)$ is a polynomial containing $2 r+2$ arbitrary constants, and $\Omega_{i, 2}(x)$ is a non-vanishing quadratic polynomial with 3-shape parameters in each subinterval defined on $I$, and $\left|\xi_{i}\right| \leq \kappa<1$, $i=1,2, \ldots, n-1$. Let $F_{i}^{(k)}(x, f)=a_{i}^{r-k}\left(\xi_{i} f+q_{i}^{(k)}(x)\right)$, where $q_{i}^{(k)}(x)$ represents the $k$ th derivative of $q_{i}(x)$ with respect to $x$. With the setting $f_{i}=d_{i}^{(0)}, d_{i}=d_{i}^{(1)}, i=1,2, \ldots, n$, if

$$
F_{i}^{(k)}\left(x_{1}, d_{1}^{(k)}\right)=d_{i}^{(k)}, \quad F_{i}^{(k)}\left(x_{n}, d_{n}^{(k)}\right)=d_{i+1}^{(k)}, \quad i=1,2, \ldots, n-1, k=0,1, \ldots, r,
$$

then the fixed point of the rational IFS $\mathcal{I}^{*}$ is the graph of the $\mathcal{C}^{r}$-rational FIF.

Proof Suppose $\mathcal{F}^{r}=\left\{h \in \mathcal{C}^{r}\left[x_{1}, x_{n}\right] \mid h\left(x_{1}\right)=f_{1}\right.$ and $\left.h\left(x_{n}\right)=f_{n}\right\}$. Now $\left(\mathcal{F}^{r}, d^{r}\right)$ is a complete metric space, where $d^{r}$ is the metric on $\mathcal{F}^{r}$ induced by the $\mathcal{C}^{r}$-norm on $\mathcal{C}^{r}\left[x_{1}, x_{n}\right]$. Define the Read-Bajraktarević operator $U$ on $\mathcal{F}^{r}$ as

$$
U h(x)=a_{i}^{r}\left\{\xi_{i} h\left(L_{i}^{-1}(x)\right)+q_{i}\left(L_{i}^{-1}(x)\right)\right\}, \quad x \in I_{i}, i=1,2, \ldots, n-1 .
$$

Since $a_{i}=\frac{x_{i+1}-x_{i}}{x_{n}-x_{1}}<1$, the conditions $\left|\xi_{i}\right| \leq \kappa<1$ and (4) imply that $U$ is a contractive operator on $\left(\mathcal{F}^{r}, d^{r}\right)$. The fixed point $\psi$ of $U$ is a fractal function that satisfies the functional equation:

$$
\psi\left(L_{i}(x)\right)=a_{i}^{r}\left\{\xi_{i} \psi(x)+q_{i}(x)\right\}, \quad x \in I, i=1,2, \ldots, n-1 .
$$

Since $\psi \in \mathcal{C}^{r}\left[x_{1}, x_{n}\right], \psi^{(k)}$ satisfies

$$
\psi^{(k)}\left(L_{i}(x)\right)=a_{i}^{r-k}\left\{\xi_{i} \psi^{(k)}(x)+q_{i}^{(k)}(x)\right\}, \quad x \in I, i=1,2, \ldots, n-1, k=0,1, \ldots, r .
$$

Using equation (4) in equation (7), we get the following system of equations for $i=$ $1,2, \ldots, n-1$ :

$$
d_{i}^{(k)}=a_{i}^{r-k}\left\{\xi_{i} d_{1}^{(k)}+q_{i}^{(k)}\left(x_{1}\right)\right\}, \quad d_{i+1}^{(k)}=a_{i}^{r-k}\left\{\xi_{i} d_{n}^{(k)}+q_{i}^{(k)}\left(x_{n}\right)\right\}, \quad \forall k=0,1, \ldots, r .
$$

When all $2 r+2$ arbitrary constants in $q_{i}(x)$ are determined from equation (8), then $\psi(x)$ exists. By using similar arguments as in [7], it can be shown that IFS $\mathcal{I}^{*}$ has a unique fixed point, and that it is the graph of the rational FIF $\psi \in \mathcal{C}^{r}\left[x_{1}, x_{n}\right]$.

\section{Rational cubic IFS}

The construction of the desired rational cubic IFS is given in Section 3.1 such that its fixed point is used for shape preservation in the sequel. The error analysis of the fixed point of rational cubic IFS with an original function is studied in Section 3.2 for convergence results. 


\subsection{Construction}

In the proposed rational cubic IFS, we assume $q_{i}(i=1,2, \ldots, n-1)$ are the rational functions with 3 -shape parameters, whose denominators are preassigned quadratics. Based on Theorem 1, with $r=1$, consider the following fixed point equation:

$$
\psi\left(L_{i}(x)\right)= \begin{cases}a_{i}\left\{\xi_{i} \psi(x)+q_{i}(x)\right\} & \text { if } \triangle_{i} \neq 0 \\ f_{i} & \text { if } \triangle_{i}=0\end{cases}
$$

where $\left|\xi_{i}\right| \leq \kappa<1$, for $i=1,2, \ldots, n-1, \Delta_{i}=\frac{f_{i+1}-f_{i}}{x_{i+1}-x_{i}}, q_{i}(x)=\frac{\Omega_{i, 1}(x)}{\Omega_{i, 2}(x)} \equiv \frac{E_{i}(\theta)}{F_{i}(\theta)}, \theta=\frac{x-x_{1}}{x_{n}-x_{1}}, x \in$ $\left[x_{1}, x_{n}\right]$,

$$
\begin{aligned}
& E_{i}(\theta)=A_{i}(1-\theta)^{3}+C_{i} \theta(1-\theta)^{2}+D_{i} \theta^{2}(1-\theta)+B_{i} \theta^{3}, \\
& F_{i}(\theta)=\alpha_{i}(1-\theta)^{2}+\gamma_{i} \theta(1-\theta)+\beta_{i} \theta^{2},
\end{aligned}
$$

$A_{i}, B_{i}, C_{i}$, and $D_{i}$ are arbitrary constants, and $\alpha_{i}, \beta_{i}$, and $\gamma_{i}$ are the shape parameters such that $\operatorname{sgn}\left(\alpha_{i}\right)=\operatorname{sgn}\left(\beta_{i}\right)=\operatorname{sgn}\left(\gamma_{i}\right)$. From this condition, it is easy to see that $F_{i}(\theta) \neq 0$ for all $\theta \in[0,1]$. To make the fixed point $\psi$ a $\mathcal{C}^{1}$-interpolant, the following Hermite interpolatory conditions are imposed:

$$
\psi\left(x_{i}\right)=f_{i}, \quad \psi\left(x_{i+1}\right)=f_{i+1}, \quad \psi^{\prime}\left(x_{i}\right)=d_{i}, \quad \psi^{\prime}\left(x_{i+1}\right)=d_{i+1} .
$$

After evaluation of $A_{i}, B_{i}, C_{i}$, and $D_{i}$ using the above Hermite interpolatory conditions, we get the desired rational cubic FIF:

$$
\psi\left(L_{i}(x)\right)= \begin{cases}a_{i} \xi_{i} \psi(x)+\frac{E_{i}(\theta)}{F_{i}(\theta)} & \text { if } \triangle_{i} \neq 0 \\ f_{i} & \text { if } \triangle_{i}=0\end{cases}
$$

where

$$
\begin{aligned}
E_{i}(\theta)= & \alpha_{i}\left(f_{i}-\xi_{i} f_{1} a_{i}\right)(1-\theta)^{3}+\left(f_{i}\left(\gamma_{i}+\alpha_{i}\right)+d_{i} \alpha_{i} h_{i}-\xi_{i}\left\{\alpha_{i} h_{i} d_{1}+f_{1} a_{i}\left(\gamma_{i}+\alpha_{i}\right)\right\}\right) \theta(1-\theta)^{2} \\
& +\left(f_{i+1}\left(\gamma_{i}+\beta_{i}\right)-d_{i+1} \beta_{i} h_{i}+\xi_{i}\left\{\beta_{i} h_{i} d_{n}-f_{n} a_{i}\left(\gamma_{i}+\beta_{i}\right)\right\}\right) \\
& \times \theta^{2}(1-\theta)+\beta_{i}\left(f_{i+1}-\xi_{i} f_{n} a_{i}\right) \theta^{3}, \\
F_{i}(\theta)= & \alpha_{i}(1-\theta)^{2}+\gamma_{i} \theta(1-\theta)+\beta_{i} \theta^{2}, \quad \theta=\frac{x-x_{1}}{x_{n}-x_{1}}, x \in\left[x_{1}, x_{n}\right] .
\end{aligned}
$$

Now it is easy to see that $\mathcal{C}^{1}$-rational cubic FIF $\psi$ is the fixed point of the following rational cubic IFS:

$$
\left\{I \times D_{1} ; w_{i}(x, f)=\left(L_{i}(x), F_{i}(x, f)\right), i=1,2, \ldots, n-1\right\},
$$

where $L_{i}(x)=a_{i} x+b_{i}, a_{i}, b_{i}$ are evaluated by using equation (1),

$$
F_{i}(x, f)= \begin{cases}a_{i} \xi_{i} f+\frac{E_{i}(\theta)}{F_{i}(\theta)} & \text { if } \triangle_{i} \neq 0, \\ f_{i} & \text { if } \triangle_{i}=0 .\end{cases}
$$

The fixed point $\psi$ of the above rational cubic IFS is unique for every fixed set of scaling factors and shape parameters. Thus by taking different sets of scaling and shape param- 
eters, we can generate an infinite number of fixed points for the above rational cubic IFS. In most applications, the derivatives $d_{i}(i=1,2, \ldots, n)$ are not given, and hence they must be calculated either from the given data or by using numerical approximation methods [24].

Remark 1 If $\xi_{i}=0$ for $i=1,2, \ldots, n-1$, then the rational cubic FIF (10) coincides with the corresponding classical rational cubic interpolation function $S$ as

$$
\begin{aligned}
S(x)= & \begin{cases}\frac{N_{1, i}}{N_{2, i}} & \text { if } \triangle_{i} \neq 0, \\
f_{i} & \text { if } \triangle_{i}=0,\end{cases} \\
N_{1, i}= & \alpha_{i} f_{i}(1-\rho)^{3}+\left\{f_{i}\left(\gamma_{i}+\alpha_{i}\right)+d_{i} \alpha_{i} h_{i}\right\} \rho(1-\rho)^{2} \\
& +\left\{f_{i+1}\left(\gamma_{i}+\beta_{i}\right)-d_{i+1} \beta_{i} h_{i}\right\} \rho^{2}(1-\rho)+f_{i+1} \rho^{3}, \\
N_{2, i}= & \alpha_{i}(1-\rho)^{2}+\gamma_{i} \rho^{2}(1-\rho)+\beta_{i} \rho^{2},
\end{aligned}
$$

described in the literature [20] with $\rho=\frac{x-x_{i}}{x_{i+1}-x_{i}}, x \in\left[x_{i}, x_{i+1}\right]$.

Remark 2 Substituting $\alpha_{i}=\beta_{i}=1$ and $\gamma_{i}=\frac{h_{i}\left(d_{i}+d_{i+1}-\xi_{i}\left(d_{1}+d_{n}\right)\right)}{f_{i+1}-f_{i}-\xi_{i} a_{i}\left(f_{n}-f_{1}\right)}$ for $i=1,2, \ldots, n-1$ in equation (10), we have

$$
\psi\left(L_{i}(x)\right)= \begin{cases}a_{i} \xi_{i} \psi(x)+\frac{E_{i}^{*}(\theta)}{F_{i}^{*}(\theta)} & \text { if } \triangle_{i} \neq 0, \\ f_{i} & \text { if } \triangle_{i}=0\end{cases}
$$

where

$$
\begin{aligned}
E_{i}^{*}(\theta)= & \left(f_{i}-\xi_{i} f_{1} a_{i}\right)(1-\theta)^{3}+\left\{\left[\frac{h_{i}\left(d_{i}+d_{i+1}-\xi_{i}\left(d_{1}+d_{n}\right)\right)}{f_{i+1}-f_{i}-\xi_{i} a_{i}\left(f_{n}-f_{1}\right)}+1\right]\right. \\
& \left.\times\left(f_{i}-\xi_{i} f_{1} a_{i}\right)+h_{i}\left(d_{i}-\xi_{i} d_{1}\right)\right\} \theta(1-\theta)^{2}+\left\{h_{i}\left(d_{i+1}-\xi_{i} d_{n}\right)\right. \\
& \left.+\left[\frac{h_{i}\left(d_{i}+d_{i+1}-\xi_{i}\left(d_{1}+d_{n}\right)\right)}{f_{i+1}-f_{i}-\xi_{i} a_{i}\left(f_{n}-f_{1}\right)}+1\right]\left(f_{i+1}-\xi_{i} f_{n} a_{i}\right)\right\} \theta(1-\theta)^{2}+\left(f_{i+1}-\xi_{i} f_{n} a_{i}\right) \theta^{3}, \\
F_{i}^{*}(\theta)= & (1-\theta)^{2}+\frac{h_{i}\left(d_{i}+d_{i+1}-\xi_{i}\left(d_{1}+d_{n}\right)\right)}{f_{i+1}-f_{i}-\xi_{i} a_{i}\left(f_{n}-f_{1}\right)} \theta(1-\theta)+\theta^{2} .
\end{aligned}
$$

After some rigorous calculations, we have found that

$$
\frac{E_{i}^{*}(\theta)}{F_{i}^{*}(\theta)}=\frac{A_{i}^{*}(1-\theta)^{2}+B_{i}^{*} \theta(1-\theta)+C_{i}^{*} \theta^{2}}{\triangle_{i}(1-\theta)^{2}+D_{i}^{*}\left(d_{i}-\xi_{i} d_{1}+d_{i+1}-\xi_{i} d_{n}\right) \theta(1-\theta)+\triangle_{i} \theta^{2}},
$$

where

$$
\begin{aligned}
& A_{i}^{*}=\triangle_{i}\left(f_{i}-\xi_{i} f_{1} a_{i}\right), \quad C_{i}^{*}=\triangle_{i}\left(f_{i+1}-\xi_{i} f_{n} a_{i}\right), \quad D_{i}^{*}=\frac{\triangle_{i}\left(x_{n}-x_{1}\right)}{f_{i+1}-f_{i}-\xi_{i} a_{i}\left(f_{n}-f_{1}\right)}, \\
& B_{i}^{*}=D_{i}^{*}\left\{a_{i}\left(f_{i} d_{i+1}+f_{i+1} d_{i}\right)-\xi_{i}\left(a_{i}\left(f_{i+1} d_{1}+f_{i} d_{n}\right)+a_{i}^{2}\left(f_{n} d_{i}+f_{1} d_{i+1}\right)\right)+\xi_{i}^{2} a_{i}^{2}\left(f_{n} d_{1}+f_{1} d_{n}\right)\right\} .
\end{aligned}
$$

Now from equation (12), we conclude that for the above choice of $\alpha_{i}, \beta_{i}$, and $\gamma_{i}$, our rational cubic FIF $\psi$ reduces to a monotonicity preserving rational quadratic FIF [25] constructed by our group. Also it is easy to verify that, if $\xi_{i}=0, \alpha_{i}=\beta_{i}=1$ and $\gamma_{i}=\frac{d_{i}+d_{i+1}}{\Delta_{i}}, i=1,2, \ldots, n-$ 1 , then the rational cubic FIF reduces to the rational quadratic function as in [23]. 


\subsection{Error analysis of fixed point of rational cubic IFS}

Theorem 2 Let $\psi$ and S, respectively, be the fixed point of rational cubic IFS (11) and the classical rational cubic function with respect to the data $\left\{\left(x_{i}, f_{i}\right), i=1,2, \ldots, n\right\}$ obtained from the original function $\Phi \in \mathcal{C}^{4}\left[x_{1}, x_{n}\right]$. Denote $\mathcal{V}=\bigotimes_{i=1}^{n-1}(-\kappa, \kappa), 0<\kappa<1, a_{\infty}=\max \left\{a_{i}\right.$ : $i=1,2, \ldots, n-1\}$. Let the shape parameters satisfy $\operatorname{sgn}\left(\alpha_{i}\right)=\operatorname{sgn}\left(\beta_{i}\right)=\operatorname{sgn}\left(\gamma_{i}\right)$. Then

$$
\|\Phi-\psi\|_{\infty} \leq \max _{1 \leq i \leq n-1}\left\{\frac{\omega_{i} h_{i}}{2 v_{i} \tau_{i}}\left(\zeta_{i}+\frac{E_{i}^{\dagger}(\Phi)}{96}\right)\right\}+\frac{\kappa a_{\infty}\left(H_{1}(h)+H_{2}(h)\right)}{1-\kappa a_{\infty}},
$$

where $E_{i}^{\dagger}(\Phi)=h_{i}^{3}\left\|\Phi^{(4)}\right\|_{\infty} A(\Phi)+16 \zeta_{i} h_{i}^{2}\left\|\Phi^{(3)}\right\|_{\infty}+24 \zeta_{i} h_{i}^{2}\left\|\Phi^{(2)}\right\|_{\infty}, A_{i}(\Phi)=\left\|\Phi^{(1)}\right\|_{\infty}+\frac{\zeta_{i}}{2}$, $\zeta_{i}=\max \left\{\left|\Phi^{\prime}\left(x_{i}\right)-d_{i}\right|,\left|\Phi^{\prime}\left(x_{i+1}\right)-d_{i+1}\right|\right\}, h=\max _{1 \leq i \leq n-1} h_{i}, v_{i}=\min _{x_{i} \leq x \leq x_{i+1}}\left|\Phi^{\prime}(x)\right|, \omega_{i}=$ $\max \left\{\alpha_{i}, \beta_{i}\right\}, \tau_{i}=\min \left\{\alpha_{i}, \beta_{i}\right\}, H_{1}(h)=\max _{1 \leq i \leq n-1}\left\{3\left(\left|f_{i}\right|+\left|f_{i+1}\right|\right)+h_{i}\left(\left|d_{i}\right|+\left|d_{i+1}\right|\right)\right\}, H_{2}(h)=$ $\max _{1 \leq i \leq n-1}\left\{3 a_{i}\left(\left|f_{1}\right|+\left|f_{n}\right|\right)+h_{i}\left(\left|d_{1}\right|+\left|d_{n}\right|\right)\right\}$.

Proof Since the coefficients of $\Omega_{i, 1}(x)$ in equation (9) depend on $\xi_{i}$, we can write $q_{i}(x)=$ $q_{i}\left(x, \xi_{i}\right)=\frac{\Omega_{i, 1}\left(x, \xi_{i}\right)}{\Omega_{i, 2}(x)} \equiv \frac{E_{i}\left(\theta, \xi_{i}\right)}{F_{i}\left(\theta, \xi_{i}\right)}$. From equation (5), the Read-Bajraktarević operator $U: \mathcal{F}^{1} \times$ $\mathcal{V} \rightarrow \mathcal{F}^{1}(c f$. Section 2 with $r=1)$ is re-written as for $x \in I_{i}$,

$$
U_{\xi} h(x)=F_{i}\left(L_{i}^{-1}(x), h \circ L_{i}^{-1}(x), \xi_{i}\right)=a_{i}\left(\xi_{i} h \circ L_{i}^{-1}(x)+q_{i}\left(L_{i}^{-1}(x), \xi_{i}\right)\right) .
$$

Let $\xi$ and $\mathbf{e}$ be the non-zero and zero vectors in $\mathcal{V}$, respectively. If $\xi_{i}=0, q_{i}(x, 0)$ is the only function of $x$ for $i=1,2, \ldots, n-1$, then the classical rational cubic interpolant $S(x)$ is the fixed point of $U_{\mathbf{e}}$. Let us assume that $\psi$ is a fixed point of a rational cubic IFS (11) associated with a non-zero scale vector $\xi$. Consequently, $\psi$ is the fixed point of $U_{\xi}$. From equation (14), it is easy to verify that $U_{\xi}$ is a contractive operator for a fixed scaling vector $\xi$ :

$$
\left\|U_{\xi} \psi-U_{\xi} S\right\|_{\infty} \leq \kappa a_{\infty}\|\psi-S\|_{\infty}
$$

Now,

$$
\begin{aligned}
\left|U_{\xi} S(x)-U_{\mathbf{e}} S(x)\right| & =a_{i}\left(\left|\xi_{i} S \circ L_{i}^{-1}(x)+q_{i}\left(L_{i}^{-1}(x), \xi_{i}\right)-q_{i}\left(L_{i}^{-1}(x), 0\right)\right|\right) \\
& \leq a_{i}\left(\left|\xi_{i}\right|\|S\|_{\infty}+\left|q_{i}\left(L_{i}^{-1}(x), \xi_{i}\right)-q_{i}\left(L_{i}^{-1}(x), 0\right)\right|\right) .
\end{aligned}
$$

Using the mean value theorem for functions of several variables, there exists $\eta=$ $\left(\eta_{1}, \eta_{2}, \ldots, \eta_{n-1}\right) \in \mathcal{V}$ such that for $i=1,2, \ldots, n-1$,

$$
q_{i}\left(L_{i}^{-1}(x), \xi_{i}\right)-q_{i}\left(L_{i}^{-1}(x), 0\right)=\frac{\partial q_{i}\left(L_{i}^{-1}(x), \eta_{i}\right)}{\partial \xi_{i}} \xi_{i}, \quad\left|\eta_{i}\right|<\left|\xi_{i}\right| .
$$

Using equation (17) in equation (16), we have

$$
\left|U_{\xi} S(x)-U_{\mathbf{e}} S(x)\right| \leq \kappa a_{\infty}\left(\|S\|_{\infty}+\left|\frac{\partial q_{i}\left(L_{i}^{-1}(x), \eta_{i}\right)}{\partial \xi_{i}}\right|\right) .
$$

Now we wish to calculate the bounds of each term in the right-hand side of equation (18). From Remark 1, it is easy to see that

$$
|S(x)| \leq \frac{S_{i, 1}(x)+S_{i, 2}(x)}{\left|S_{i, d}(\rho)\right|}
$$


where

$$
\begin{aligned}
& S_{i, 1}(x)=\left|\alpha_{i}\right|\left|f_{i}\right|(1-\rho)^{3}+\left\{\left|f_{i}\right|\left(\left|\gamma_{i}\right|+\left|\alpha_{i}\right|\right)+\left|d_{i}\right|\left|\alpha_{i}\right| h_{i}\right\} \rho(1-\rho)^{2}, \\
& S_{i, 2}(x)=\left\{\left|f_{i+1}\right|\left(\left|\gamma_{i}\right|+\left|\beta_{i}\right|\right)+\left|d_{i+1}\right|\left|\beta_{i}\right| h_{i}\right\} \rho^{2}(1-\rho)+\left|f_{i+1}\right| \rho^{3}, \\
& S_{i, d}(\rho)=\alpha_{i}(1-\rho)^{2}+\gamma_{i} \rho^{2}(1-\rho)+\beta_{i} \rho^{2} .
\end{aligned}
$$

Since $\operatorname{sgn}\left(\alpha_{i}\right)=\operatorname{sgn}\left(\beta_{i}\right)=\operatorname{sgn}\left(\gamma_{i}\right)$ for $i=1,2, \ldots, n-1$, we have

$$
\left|S_{i, d}(\rho)\right| \geq \max \left\{\left|\alpha_{i}\right|(1-\rho)^{2},\left|\gamma_{i}\right| \rho^{2}(1-\rho),\left|\beta_{i}\right| \rho^{2}\right\} \quad \text { for each } \rho \in[0,1]
$$

Now using equation (20) in equation (19), we get $|S(x)| \leq 3\left|f_{i}\right|+3\left|f_{i+1}\right|+h_{i}\left(\left|d_{i}\right|+\left|d_{i+1}\right|\right)$.

Since the above inequality is true for all $i=1,2, \ldots, n-1$, we get the following estimation:

$$
\|S\|_{\infty} \leq H_{1}(h)
$$

Since $\Omega_{i, 2}(x) \equiv F_{i}(\theta)$ is independent of $\xi_{i}$, it easy to see that

$$
\frac{\partial q_{i}\left(L_{i}^{-1}(x), \gamma_{i}\right)}{\partial \alpha_{i}}=\frac{-\left(Q_{i, 1}(\theta)+Q_{i, 2}(\theta)\right)}{\Omega_{i, 2}\left(L_{i}^{-1}(x)\right)}, \quad \theta=\frac{L_{i}^{-1}(x)-x_{1}}{x_{n}-x_{1}}, x \in\left[x_{i}, x_{i+1}\right],
$$

where

$$
\begin{aligned}
& Q_{i, 1}(\theta)=\alpha_{i} f_{1} a_{i}(1-\theta)^{3}+\left\{\alpha_{i} h_{i} d_{1}+f_{1} a_{i}\left(\gamma_{i}+\alpha_{i}\right)\right\} \theta(1-\theta)^{2}, \\
& Q_{i, 2}(\theta)=\left\{\beta_{i} h_{i} d_{n}-f_{n} a_{i}\left(\gamma_{i}+\beta_{i}\right)\right\} \theta^{2}(1-\theta)+\beta_{i} f_{n} a_{i} \theta^{3} .
\end{aligned}
$$

By using similar arguments as used in the estimation of $\|S\|_{\infty}$, we have found that

$$
\left|\frac{\partial q_{i}\left(L_{i}^{-1}(x), \eta_{i}\right)}{\partial \xi_{i}}\right| \leq H_{2}(h), \quad i=1,2, \ldots, n-1 .
$$

By using equations (21) and (22) in equation (18), we have

$$
\left|U_{\xi} S(x)-U_{\mathbf{e}} S(x)\right| \leq \kappa a_{\infty}\left(H_{1}(h)+H_{2}(h)\right) .
$$

Since the above inequality is true for $i=1,2, \ldots, n-1$,

$$
\left\|U_{\xi} S-U_{\mathbf{e}} S\right\|_{\infty} \leq \kappa a_{\infty}\left(H_{1}(h)+H_{2}(h)\right) .
$$

Combining equations (15) and (23) with the inequality

$$
\|\psi-S\|_{\infty}=\left\|U_{\xi} \psi-U_{\mathbf{e}} S\right\|_{\infty} \leq\left\|U_{\xi} \psi-U_{\xi} S\right\|_{\infty}+\left\|U_{\xi} S-U_{\mathbf{e}} S\right\|_{\infty}
$$

we get

$$
\|\psi-S\|_{\infty} \leq \frac{|\xi|_{\infty} a_{\infty}\left(H_{1}(h)+H_{2}(h)\right)}{1-\kappa a_{\infty}} .
$$

From equation (24), it is evident that for $\xi_{i}=0, i=1,2, \ldots, n-1$, the fixed point of rational cubic IFS (11) coincides with the corresponding classical rational cubic interpolant. 
Since the original function $\Phi \in \mathcal{C}^{4}\left[x_{1}, x_{n}\right]$, it is known that [20]

$$
\|\Phi-S\|_{\infty} \leq \max _{1 \leq i \leq n-1}\left\{\frac{\omega_{i} h_{i}}{2 v_{i} \tau_{i}}\left(\zeta_{i}^{*}+\frac{E_{i}^{\dagger}(\Phi)}{96}\right)\right\}
$$

Therefore, using equations (24)-(25) together with the inequality $\|\Phi-\psi\|_{\infty} \leq \| f-$ $S\left\|_{\infty}+\right\| S-\psi \|_{\infty}$, we get the bound for $\|\Phi-\psi\|_{\infty}$, and it completes the proof of theorem.

Corollary 1 (Convergence results) Assume that $H_{1}(h)$ and $H_{2}(h)$ are bounded as $h \rightarrow 0^{+}$. Then we have the following results:

(i) Since $a_{\infty}=\frac{h}{x_{n}-x_{1}}$, we conclude from equation (13) that the fixed point of rational cubic IFS equation (11) converges uniformly to the original function $\Phi$ as $h \rightarrow 0$.

(ii) Again from the error estimation (13), $O\left(h^{p}\right)(p=2,3,4)$ convergence can be obtained if the derivative values are available such that $\zeta_{i}=O\left(h_{i}^{p-1}\right)(p=2,3,4)$, and the scaling factors are chosen as $\left|\xi_{i}\right| \leq \kappa a_{i}^{p-1}(p=2,3,4)$ for $i=1,2, \ldots, n-1$.

\section{Positivity preserving rational cubic FIF}

The $\mathcal{C}^{1}$-rational cubic fractal interpolation function developed in Section 3 has deficiencies as far as the positivity preserving issue is concerned. Because of the recursive nature of FIFs, we assume all the scaling factors are non-negative so that it is easy to derive the sufficient conditions for a positive fixed point of the rational cubic IFS (11). It requires one to assign appropriate restrictions on the scaling factors $\xi_{i}$ and shape parameters $\alpha_{i}$, $\beta_{i}$ and $\gamma_{i}$, for $i=1,2, \ldots, n-1$, so that the positivity feature of a given set of positive data is preserved in the fixed point of the rational cubic IFS (11). In Section 4.1, the suitable restrictions are developed on the scaling factors and shape parameters for a positivity preserving $\mathcal{C}^{1}$-rational cubic spline FIF. The importance of suitable restrictions on the rational IFS parameters is illustrated in Section 4.2.

\subsection{Restrictions on IFS parameters for positivity}

Theorem 3 Let $\left\{\left(x_{i}, f_{i}, d_{i}\right), i=1,2, \ldots, n\right\}$ be a given positive data. If

(i) the scaling factors $\xi_{i}, i=1,2, \ldots, n-1$, are selected as

$$
\xi_{i} \in \begin{cases}{\left[0, \min \left\{\frac{f_{i}}{a_{i} f_{1}}, \frac{f_{i+1}}{a_{i} f_{n}}\right\}\right]} & \text { if } \min \left\{\frac{f_{i}}{a_{i} f_{1}}, \frac{f_{i+1}}{a_{i j} f_{n}}\right\}<\kappa, \\ {[0, \kappa]} & \text { if } \min \left\{\frac{f_{i}}{a_{i} f_{1}}, \frac{f_{i+1}}{a_{i j} f_{n}}\right\} \geq \kappa,\end{cases}
$$

(ii) with $\gamma_{i}>0$, the shape parameters $\alpha_{i}, \beta_{i}, i=1,2, \ldots, n-1$, are chosen as

$$
\begin{cases}\alpha_{i}>0 & \text { if } \lambda_{i} \geq 0 \\ \alpha_{i} \in\left(0, \frac{-\gamma_{i} s_{i}^{*}}{\lambda_{i}}\right) & \text { if } \lambda_{i}<0 \\ \beta_{i}>0 & \text { if } \mu_{i} \geq 0 \\ \beta_{i} \in\left(0, \frac{-\gamma_{i} p_{i}^{*}}{\mu_{i}}\right) & \text { if } \mu_{i}<0\end{cases}
$$

where $p_{i}^{*}=f_{i+1}-\xi_{i} f_{n} a_{i}, s_{i}^{*}=f_{i}-\xi_{i} f_{1} a_{i}, \lambda_{i}=f_{i}+d_{i} h_{i}-\xi_{i}\left(h_{i} d_{1}+f_{1} a_{i}\right)$, $\mu_{i}=f_{i+1}-d_{i+1} h_{i}-\xi_{i}\left(f_{n} a_{i}-h_{i} d_{n}\right)$, then for fixed $\xi_{i}, \alpha_{i}, \beta_{i}, \gamma_{i}(i=1,2, \ldots, n-1)$, the unique fixed point $\psi$ of the rational IFS (11) is positive. 
Proof From equation (10), we have

$$
\psi\left(L_{i}(x)\right)=a_{i} \xi_{i} \psi(x)+\frac{E_{i}(\theta)}{F_{i}(\theta)} .
$$

It is easy to verify that using equation (2), if $\xi_{i} \geq 0, i=1,2, \ldots, n-1$, the sufficient conditions for $\psi\left(L_{i}(x)\right)>0$ for all $x \in\left[x_{1}, x_{n}\right]$ are $\frac{E_{i}(\theta)}{F_{i}(\theta)}>0$ for all $\theta \in[0,1], i=1,2, \ldots, n-1$. If we assume $\alpha_{i}>0, \beta_{i}>0$, and $\gamma_{i}>0$, then it is easy to see that $F_{i}(\theta)>0$ for any $\theta \in[0,1]$. Thus the initial conditions on the scaling factor and shape parameters are $\xi_{i} \geq 0$, and $\alpha_{i}>0, \beta_{i}>0, \gamma_{i} \geq 0$, respectively, for $i=1,2, \ldots, n-1$. Including the initial conditions on the shape parameters, we have $\frac{E_{i}(\theta)}{F_{i}(\theta)}>0 \Leftrightarrow E_{i}(\theta)>0 \forall \theta \in[0,1], i=1,2, \ldots, n-1$. Thus our problem reduces to finding conditions on the scaling factors and shape parameters for which $E_{i}(\theta)>0$ for all $\theta \in[0,1]$. From equation $(10), E_{i}(\theta)$ is re-written as

$$
E_{i}(\theta)=p_{i} \theta^{3}+q_{i} \theta^{2}+r_{i} \theta+s_{i}
$$

where

$$
\begin{aligned}
& p_{i}=\gamma_{i}\left(f_{i}-f_{i+1}\right)+h_{i}\left(\alpha_{i} d_{i}+\beta_{i} d_{i+1}\right), \\
& q_{i}=\left(\alpha_{i}-2 \gamma_{i}\right) f_{i}+\left(\gamma_{i}+\beta_{i}\right) f_{i+1}-h_{i}\left(2 \alpha_{i} d_{i}+\beta_{i} d_{i+1}\right), \\
& r_{i}=\left(\gamma_{i}-2 \alpha_{i}\right) f_{i}+\alpha_{i} h_{i} d_{i}, \quad s_{i}=\alpha_{i} f_{i} .
\end{aligned}
$$

By substituting $\theta=\frac{s}{s+1}$ in equation (28), $E_{i}(\theta)>0$ for all $\theta \in[0,1]$ is equivalent to $\Omega_{i}(s)=$ $p_{i}^{*} s^{3}+q_{i}^{*} s^{2}+r_{i}^{*} s+s_{i}^{*}>0$ for all $s \geq 0$, where $p_{i}^{*}=p_{i}+q_{i}+r_{i}+s_{i}, q_{i}^{*}=q_{i}+2 r_{i}+3 s_{i}, r_{i}^{*}=r_{i}+3 s_{i}$, $s_{i}^{*}=s_{i}$.

From [14], we have $\Omega_{i}(s)>0$ for all $s \geq 0$ if and only if $\left(p_{i}^{*}, q_{i}^{*}, r_{i}^{*}, s_{i}^{*}\right) \in R_{1} \cup R_{2}$, where

$$
\begin{aligned}
R_{1}= & \left\{\left(p_{i}^{*}, q_{i}^{*}, r_{i}^{*}, s_{i}^{*}\right): p_{i}^{*}>0, q_{i}^{*}>0, r_{i}^{*}>0, s_{i}^{*}>0\right\}, \\
R_{2}= & \left\{\left(p_{i}^{*}, q_{i}^{*}, r_{i}^{*}, s_{i}^{*}\right): p_{i}^{*}>0, s_{i}^{*}>0,\right. \\
& \left.4 p_{i}^{*} r_{i}^{* 3}+4 s_{i}^{*} q_{i}^{* 3}+27 p_{i}^{* 2} s_{i}^{* 2}-18 p_{i}^{*} q_{i}^{*} r_{i}^{*} s_{i}^{*}-q_{i}^{* 2} r_{i}^{* 2}>0\right\} .
\end{aligned}
$$

Let $\left(p_{i}^{*}, q_{i}^{*}, r_{i}^{*}, s_{i}^{*}\right) \in R_{1}$, then we have $p_{i}^{*}>0, s_{i}^{*}>0, q_{i}^{*}=\beta_{i} \mu_{i}+\gamma_{i} p_{i}^{*}>0, r_{i}^{*}=\alpha_{i} \lambda_{i}+\gamma_{i} s_{i}^{*}>0$. Now $p_{i}^{*}>0, s_{i}^{*}>0$ if and only if $\xi_{i}<\frac{f_{i}}{f_{1} a_{i}}, \xi_{i}<\frac{f_{i+1}}{f_{n} a_{i}}$, respectively. Hence, the restriction on the scaling factor $\xi_{i}$ is

$$
\xi_{i}<\min \left\{\frac{f_{i}}{f_{1} a_{i}}, \frac{f_{i+1}}{f_{n} a_{i}}\right\} \text {. }
$$

If $\lambda_{i} \geq 0, r_{i}^{*}=\alpha_{i} \lambda_{i}+\gamma_{i} s_{i}^{*}>0$ is true from equation (29), and in this case $\alpha_{i}>0$ can be chosen arbitrarily. Otherwise, $\lambda_{i}<0$, we have $r_{i}^{*}=\alpha_{i} \lambda_{i}+\gamma_{i} s_{i}^{*}>0 \Leftrightarrow \alpha_{i}<\frac{-\gamma_{i} s_{i}^{*}}{\lambda_{i}}$. Similarly $q_{i}^{*}=\beta_{i} \mu_{i}+\gamma_{i} p_{i}^{*}>0$ is true when (i) $\mu_{i} \geq 0, \beta_{i}>0$ arbitrary (ii) $\mu_{i}<0, \beta_{i}<\frac{-\gamma_{i} p_{i}^{*}}{\mu_{i}}$. Another set of restrictions on $\xi_{i}, \alpha_{i}, \beta_{i}$, and $\gamma_{i}$ can be derived if $\left(p_{i}^{*}, q_{i}^{*}, r_{i}^{*}, s_{i}^{*}\right) \in R_{2}$. But we have not considered it here due to the complexity involved in the calculations. The above discussions yield equation (27).

Therefore, $E_{i}(\theta) \geq 0$ whenever equations (29) and (27) are true. Now it is easy to see that the fixed point of the rational cubic IFS (11) is positive if the scaling factors and shape parameters involved in the IFS (11) satisfy equations (26) and (27), respectively. 


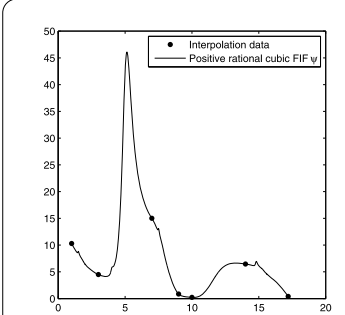

(a) The original function $\Phi$.

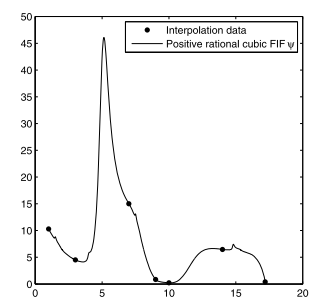

(d) Effects of $\alpha_{6}$ in Figure 1(a).

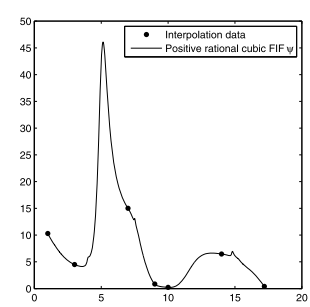

(b) Effects of $\xi_{1}$ in Figure 1(a).

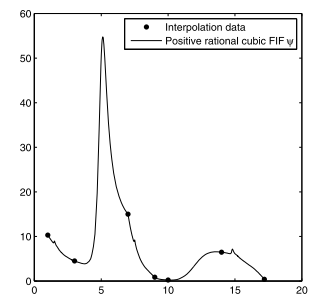

(e) Effects of $\beta_{3}$ in Figure 1(c).

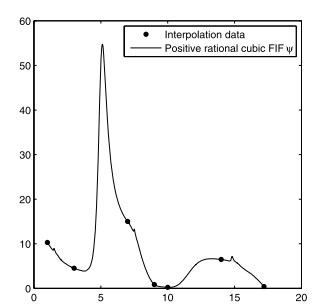

(c) Effects of $\xi_{2}$ in Figure 1(a).

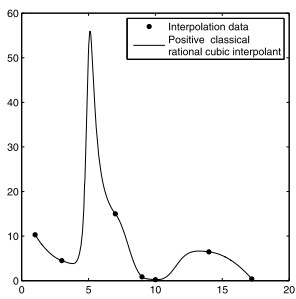

(f) Classical rational cubic interpolant

Figure 1 Illustration of positive rational fractal interpolants with shape parameters.

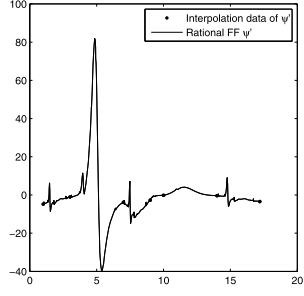

(a) Derivative of original function $\Phi$.

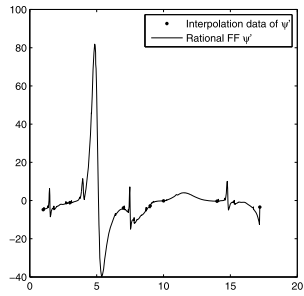

(d) Derivative of rational cubic FIF in Figure 1(d).

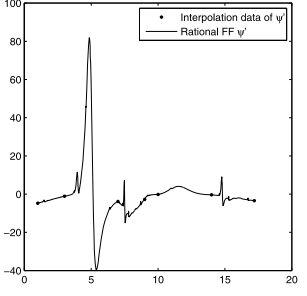

(b) Derivative of rational cubic FIF in Figure 1(b).

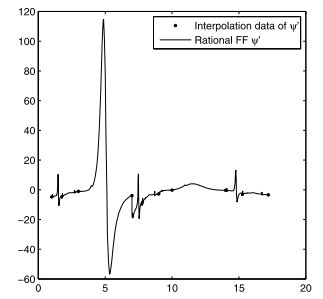

(e) Derivative of rational cubic FIF in Figure 1(e).

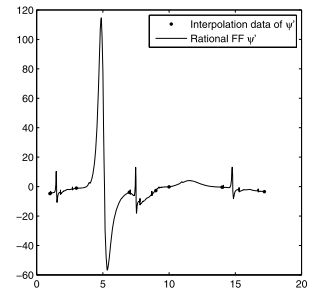

(c) Derivative of rational cubic FIF in Figure 1(c).

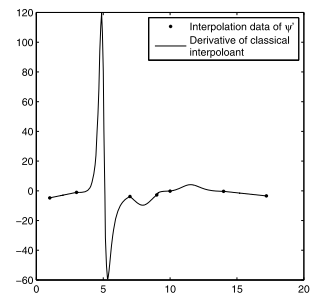

(f) Derivative of classical rational cubic interpolant in Figure 1(f).

Figure 2 Derivatives of positive rational fractal interpolants and classical interpolant.

\subsection{Examples and discussion}

In order to demonstrate the positive interpolation using our rational cubic IFS, consider the positive data set $\{(1,10.3),(3,4.5),(7,15),(9,0.85),(10,0.23),(14,6.47),(17.2,0.4)\}$, which is taken from a function (see Figure 1(a)) with an irregular derivative function as described in Figure 2(a). Such type of data arises in the motion of a single inverted pendulum in the field of non-linear control theory [22]. The position of the cart can be taken as smooth and positive in a short time interval with an irregular cart velocity. To ap- 
Table 1 Rational IFS parameters for positive fractal interpolants

\begin{tabular}{lll}
\hline Figure & Scaling factors & Shape parameters \\
\hline 1 (a) & $\xi_{1}=0.123, \xi_{2}=0.107, \xi_{3}=0.179$, & $\beta_{i}=\gamma_{i}=1, i=1(1) 6$, \\
& $\xi_{4}=0.005, \xi_{5}=0.005, \xi_{6}=0.124$ & $\alpha_{i}=1, i=1,2,3,6, \alpha_{4}=0.445, \alpha_{5}=0.512$ \\
$1(\mathrm{~b})$ & $\xi_{1}=\mathbf{0 . 0 1}, \xi_{2}=0.107, \xi_{3}=0.179$, & $\beta_{i}=\gamma_{i}=1, i=1(1) 6$, \\
& $\xi_{4}=0.005, \xi_{5}=0.005, \xi_{6}=0.124$ & $\alpha_{i}=1, i=1,2,3,6, \alpha_{4}=0.445, \alpha_{5}=0.512$ \\
$1(\mathrm{c})$ & $\xi_{1}=0.123, \xi_{2}=0.107, \xi_{3}=\mathbf{0 . 0 1}$, & $\beta_{i}=\gamma_{i}=1, i=1(1) 6$, \\
& $\xi_{4}=0.005, \xi_{5}=0.005, \xi_{6}=0.124$ & $\alpha_{i}=1, i=1,2,3,6, \alpha_{4}=0.445, \alpha_{5}=0.512$ \\
1 (d) & $\xi_{1}=0.123, \xi_{2}=0.107, \xi_{3}=0.179$, & $\beta_{i}=\gamma_{i}=1, i=1(1) 6$, \\
& $\xi_{5}=0.005, \xi_{6}=0.124$ & $\xi_{4}=0.005, \alpha_{i}=1, i=1,2,3$, \\
& & $\alpha_{4}=0.445, \alpha_{5}=0.512, \alpha_{6}=\mathbf{1 0} \mathbf{9}$ \\
$1(\mathrm{e})$ & $\xi_{1}=0.123, \xi_{2}=0.107, \xi_{3}=\mathbf{0 . 0 1}$, & $\beta_{i}=\gamma_{i}=1, i \in\{1,2,4,5,6\}, \gamma_{3}=1, \beta_{3}=\mathbf{1 0}^{\mathbf{3}}$, \\
& $\xi_{4}=0.005, \xi_{5}=0.005, \xi_{6}=0.124$ & $\alpha_{i}=1, i=1,2,3,6, \alpha_{4}=0.445, \alpha_{5}=0.512$ \\
1 (f) & $\xi_{1}=\xi_{2}=\xi_{3}=\xi_{4}=\xi_{5}=\xi_{6}=\xi_{7}=\mathbf{0}$ & $\beta_{i}=\gamma_{i}=1, i=1(1) 6$, \\
& & $\alpha_{i}=1, i=1,2,3,6, \alpha_{4}=0.445, \alpha_{5}=0.512$ \\
\hline
\end{tabular}

proximate such data, we have employed the rational cubic IFS (11). The derivative values at the knots are approximated by the arithmetic mean method [24] as $d_{1}=-4.7417$, $d_{2}=-1.0583, d_{3}=-3.8417, d_{4}=-2.7717, d_{5}=-0.184, d_{6}=-0.3605$, and $d_{7}=-3.4333$. The scaling factors are constrained as $\xi_{1} \in[0,0.1235], \xi_{2} \in[0,0.1079], \xi_{3} \in[0,0.1798]$, $\xi_{4} \in[0,0.0051], \xi_{5} \in[0,0.0055], \xi_{6} \in[0,0.1241]$ by equation (26) with a choice of $\kappa=0.9$.

The IFS parameters of the original function are given in Table 1, and aesthetic modifications are illustrated by varying the scaling factors and shape parameters. In order to explain the sensitiveness of a rational cubic FIF with respect to the scaling factors, we have taken a fixed set of shape parameters in the construction of Figures 1(b)-(c), see Table 1. By comparing Figure 1(b) with Figure 1(a), we observe that the fractal curve pertaining to the first subinterval $\left[x_{1}, x_{2}\right]$ converges to a convex shape as $\xi_{1} \rightarrow 0^{+}$, and changes in other subintervals are negligible. By comparing the shapes of Figure 1(a) and Figure 1(c), we notice perceptible variations in the second subinterval, and variations in other subintervals are negligible. By comparing Figures 1(d)-(e) with Figure 1(a) and Figure 1(c), respectively, we can observe the sensitivity of the positive FIF with respect to its shape parameters. Finally, we have constructed the classical rational cubic interpolant in Figure 1(f) with the zero scaling vector. From the above discussion, we conclude that the effects due to the scaling factors $\xi_{1}, \xi_{2}$, and shape parameters $\alpha_{6}, \beta_{3}$ are very local in nature for the given positive data set.

From equations (4) and (10), $\psi^{\prime}$ interpolates the data $\left\{\left(x_{i}, d_{i}\right): i=1,2, \ldots, n\right\}$. In this example, the interpolation data for $\psi^{\prime}$ is $\{(1,-4.7417),(3,-1.0583),(7,-3.8417),(9,-2.7717)$, $(10,-0.184),(14,-0.3605),(17.2,-3.4333)\}$. The derivative functions of rational cubic FIFs in Figures 1(a)-(e) are constructed in Figures 2(a)-(e), respectively, and they are typically irregular fractal functions close to a continuous function, but at least they differ from a piecewise differentiable function. We have calculated the uniform errors between this original function $\Phi$ in Figure 1(a) and the rational cubic FIFs in Figures 1(b)-(f) (see Table 2). Also we have calculated the uniform errors between their derivatives (see Table 2). The effects of the scaling factors $\xi_{1}$ are very prominent in the first subinterval of Figure 1(b), but they also render major effects in its derivative (see Figure 2(b) and Table 2). The effects of the scaling factor $\xi_{2}$ are prominent in the second and third subintervals of Figure 2(c) in comparison with Figure 2(a). Hence the scaling factor $\xi_{2}$ is moderately local in nature in the derivative of rational cubic FIF. The shape parameters $\alpha_{6}$ and $\beta_{3}$ produce similar effects 
Table 2 Uniform errors between $\Phi$ and rational fractal interpolants, and their derivatives

\begin{tabular}{llll}
\hline $\begin{array}{l}\text { Rational cubic } \\
\text { FIF }\end{array}$ & $\begin{array}{l}\text { Uniform distance with } \\
\text { Figure 1(a) }\end{array}$ & $\begin{array}{l}\text { Derivative of rational } \\
\text { cubic FIF }\end{array}$ & $\begin{array}{l}\text { Uniform distance with } \\
\text { Figure 2(a) }\end{array}$ \\
\hline Figure 1(b) & 0.0401 & Figure 2(b) & 0.5042 \\
Figure 1(c) & 0.1901 & Figure 2(c) & 1.7129 \\
Figure 1(d) & 0.1646 & Figure 2(d) & 1.8654 \\
Figure 1(e) & 0.0379 & Figure 2(e) & 1.0421 \\
Figure 1(f) & 0.3154 & Figure 2(f) & 2.9245 \\
\hline
\end{tabular}

(see the corresponding figures and Table 2). The rational fractal functions in Figures 2(a)(e) are irregular in nature over the interval $\left[x_{1}, x_{7}\right]$, whereas the derivative of a classical interpolant is piecewise differentiable in the interval $\left[x_{1}, x_{7}\right]$ (see Figure 2(f)). Comparing the uniform distances in Table 2, if the original function is $\mathcal{C}^{1}$-smooth and positive but its derivative is very irregular, then our rational cubic IFS is an ideal tool for approximating such a function instead of the classical rational cubic interpolant whose derivative is a piecewise smooth function.

\section{Monotonicity preserving rational cubic FIF}

The fixed point $\psi$ of a rational cubic IFS may not preserve the monotonic feature of a given set of monotonic data. For an automatic generation of rational IFS parameters, we restrict them in Section 5.1, and the results are implemented in Section 5.2 through suitable examples.

\subsection{Restrictions on IFS parameters for monotonicity}

Theorem 4 Let $\left\{\left(x_{i}, f_{i}, d_{i}\right), i=1,2, \ldots, n\right\}$ be a given monotonic data. Let the derivative values satisfy the necessary conditions for monotonicity, namely

$$
\begin{cases}d_{i}=d_{i+1}=0 & \text { for } \triangle_{i}=0 \\ \operatorname{sgn}\left(d_{i}\right)=\operatorname{sgn}\left(d_{i+1}\right)=\operatorname{sgn}\left(\triangle_{i}\right) & \text { for } \triangle_{i} \neq 0\end{cases}
$$

If (i) the scaling factors $\xi_{i}, i=1,2, \ldots, n-1$, are chosen as

$$
\xi_{i} \in \begin{cases}{\left[0, \min \left\{\frac{d_{i+1}}{d_{n}}, \frac{d_{i}}{d_{1}}\right\}\right]} & \text { if } \min \left\{\frac{d_{i+1}}{d_{n}}, \frac{d_{i}}{d_{1}}\right\}<\min \left\{\frac{\Delta_{i}\left(x_{n}-x_{1}\right)}{f_{n}-f_{1}}, \kappa\right\}, \\ {\left[0, \min \left\{\frac{\Delta_{i}\left(x_{n}-x_{1}\right)}{f_{n}-f_{1}}, \kappa\right\}\right]} & \text { if } \min \left\{\frac{d_{i+1}}{d_{n}}, \frac{d_{i}}{d_{1}}\right\} \geq \min \left\{\frac{\Delta_{i}\left(x_{n}-x_{1}\right)}{f_{n}-f_{1}}, \kappa\right\},\end{cases}
$$

(ii) the shape parameters $\alpha_{i}, \beta_{i}$, and $\gamma_{i}, i=1,2, \ldots, n-1$, are selected as

$$
\operatorname{sgn}\left(\alpha_{i}\right)=\operatorname{sgn}\left(\beta_{i}\right), \quad \text { and } \quad \gamma_{i}=\frac{\alpha_{i}\left(d_{i}-\xi_{i} d_{1}\right)+\beta_{i}\left(d_{i+1}-\xi_{i} d_{n}\right)}{\triangle_{i}-\xi_{i} \frac{f_{n}-f_{1}}{x_{n}-x_{1}}}
$$

then for a fixed $\xi, \alpha_{i}, \beta_{i}, \gamma_{i}(i=1,2, \ldots, n-1)$, the unique fixed point $\psi$ of the rational cubic IFS (11) is monotonic in nature.

Proof Differentiating equation (10) with respect to $x$, after some mathematical manipulations, we get

$$
\psi^{\prime}\left(L_{i}(x)\right)=\xi_{i} \psi^{\prime}(x)+\frac{\sum_{j=1}^{5} A_{j, i} \theta^{j-1}(1-\theta)^{5-j}}{\left(F_{i}(\theta)\right)^{2}}
$$


where

$$
\begin{aligned}
A_{1, i}= & \left(d_{i}-\xi_{i} d_{1}\right) \alpha_{i}^{2}, \quad A_{5, i}=\left(d_{i+1}-\xi_{i} d_{n}\right) \beta_{i}^{2}, \\
A_{2, i}= & 2 \alpha_{i}\left[\left(\gamma_{i}+\beta_{i}\right) \triangle_{i}-\beta_{i} d_{i}\right]-2 \xi_{i} \alpha_{i}\left[\left(\gamma_{i}+\beta_{i}\right) \frac{f_{n}-f_{1}}{x_{n}-x_{1}}-\beta_{i} d_{1}\right], \\
A_{3, i}= & {\left[3 \alpha_{i} \beta_{i}+\left(\gamma_{i}+\beta_{i}\right)\left(\gamma_{i}+\alpha_{i}\right)\right] \triangle_{i}-\left[\beta_{i} d_{i+1}\left(\gamma_{i}+\alpha_{i}\right)+\alpha_{i} d_{i}\left(\gamma_{i}+\beta_{i}\right)\right] } \\
& -\xi_{i}\left[3 \alpha_{i} \beta_{i}+\left(\gamma_{i}+\beta_{i}\right)\left(\gamma_{i}+\alpha_{i}\right) \frac{f_{n}-f_{1}}{x_{n}-x_{1}}-\left\{\alpha_{i} d_{1}\left(\gamma_{i}+\beta_{i}\right)+\beta_{i} d_{n}\left(\gamma_{i}+\alpha_{i}\right)\right\}\right], \\
A_{4, i}= & 2 \beta_{i}\left[\left(\gamma_{i}+\alpha_{i}\right) \triangle_{i}-\alpha_{i} d_{i+1}\right]-2 \xi_{i} \beta_{i}\left[\left(\gamma_{i}+\alpha_{i}\right) \frac{f_{n}-f_{1}}{x_{n}-x_{1}}-\alpha_{i} d_{n}\right] .
\end{aligned}
$$

Due to the recursive nature of rational fractal function (33), the necessary conditions (30) are not sufficient to ensure the monotonicity of fixed point $\psi$ of the rational cubic IFS (11). We impose additional restrictions on the scaling factors $\xi_{i}$, and shape parameters $\alpha_{i}, \beta_{i}$, and $\gamma_{i}, i=1,2, \ldots, n-1$, so that these conditions together with the necessary conditions (30) yield the monotonic feature of the fixed point $\psi$ of the IFS (11).

\section{Case I: Monotonically increasing data}

Suppose $\left\{\left(x_{i}, f_{i}\right), i=1,2, \ldots, n\right\}$ is a given monotonically increasing data set. Due to the recursive nature of IFS and equation (2), it is assumed that all the scaling factors $\xi_{i}$, $i=1,2, \ldots, n-1$, are non-negative for a monotonic fixed point of rational cubic IFS (11). For $\triangle_{i}=0, \psi\left(L_{i}(x)\right)=f_{i}$, which is monotone on $\left[x_{i}, x_{i+1}\right]$ (choose $\left.\xi_{i}=0\right)$. Otherwise for $\triangle_{i}>0$, the sufficient conditions for the monotonicity of the fixed point of rational cubic IFS (11) are $A_{j, i} \geq 0, j=1,2, \ldots, 5$. From equation (33),

$$
A_{1, i} \geq 0 \quad \Leftrightarrow \quad\left(d_{i}-\xi_{i} d_{1}\right) \alpha_{i}^{2} \geq 0 \quad \Leftrightarrow \quad d_{i}-\xi_{i} d_{1} \geq 0 \quad \Leftrightarrow \quad \xi_{i} \leq \frac{d_{i}}{d_{1}} .
$$

Similarly,

$$
A_{5, i} \geq 0 \quad \Leftrightarrow \quad \xi_{i} \leq \frac{d_{i+1}}{d_{n}} .
$$

From equation (33), $A_{2, i}$ is re-written as

$$
A_{2, i}=2 \alpha_{i}\left(\gamma_{i}+\beta_{i}\right)\left(\triangle_{i}-\xi_{i} \frac{f_{n}-f_{1}}{x_{n}-x_{1}}\right)+2 \alpha_{i} \beta_{i}\left(d_{i}-\xi_{i} d_{1}\right) .
$$

Without loss of generality, assume that

$$
\triangle_{i}-\xi_{i} \frac{f_{n}-f_{1}}{x_{n}-x_{1}}>0
$$

i.e., $\xi_{i}<\frac{\Delta_{i}\left(x_{n}-x_{1}\right)}{f_{n}-f_{1}}$. We search for sufficient conditions that make $A_{2, i} \geq 0$. For this purpose, we make each term in $A_{2, i}$ non-negative. The selection of $\xi_{i}$ with respect to equations (34) and (36) gives $d_{i}-\xi_{i} d_{1} \geq 0$ and $\Delta_{i}-\xi_{i} \frac{f_{n}-f_{1}}{x_{n}-x_{1}}>0$, respectively. Now it remains to make $\alpha_{i}\left(\gamma_{i}+\beta_{i}\right) \geq 0$ and $\alpha_{i} \beta_{i} \geq 0$. In these two inequalities, the product of the shape parameters is involved. Therefore these inequalities are true if we restrict the shape parameters $\alpha_{i}, \beta_{i}$, and $\gamma_{i}, i=1,2, \ldots, n-1$, respectively, as in equation (32). 
Justification for equation (32)

Let $\operatorname{sgn}\left(\alpha_{i}\right)=\operatorname{sgn}\left(\beta_{i}\right)$ be negative, then from equations (32) and (34)-(36), we can conclude that $\operatorname{sgn}\left(\gamma_{i}\right)$ is negative. Therefore, $\alpha_{i}\left(\gamma_{i}+\beta_{i}\right) \geq 0$ and $\alpha_{i} \beta_{i} \geq 0$. Similarly, it can be shown that $\operatorname{sgn}\left(\alpha_{i}\right)=\operatorname{sgn}\left(\beta_{i}\right)$ being positive gives similar results.

The above discussion led to the following procedure to make $A_{2, i} \geq 0$ : first choose the scaling factors with respect to equations (34)-(36), then select the shape parameters according to equation (32).

Again from equation (33), $A_{4, i}$ is re-arranged as $A_{4, i}=2 \beta_{i}\left(\gamma_{i}+\alpha_{i}\right)\left(\triangle_{i}-\xi_{i} \frac{f_{n}-f_{1}}{x_{n}-x_{1}}\right)+$ $2 \alpha_{i} \beta_{i}\left(d_{i+1}-\xi_{i} d_{n}\right)$. Similarly, it is easy to verify that equations (32) and (34)-(36) are sufficient for $A_{4, i} \geq 0$. For simplicity, denote $d_{i}^{*}=d_{i}-\xi_{i} d_{1}, d_{i+1}^{*}=d_{i+1}-\xi_{i} d_{n}, \triangle_{i}^{*}=\triangle_{i}-\xi_{i} \frac{f_{n}-f_{1}}{x_{n}-x_{1}}$. From equation (33), and with the above notations, $A_{3, i}$ is re-written as

$$
\begin{aligned}
A_{3, i} & =\left[3 \alpha_{i} \beta_{i}+\left(\gamma_{i}+\beta_{i}\right)\left(\gamma_{i}+\alpha_{i}\right)\right] \triangle_{i}^{*}-\alpha_{i}\left(\gamma_{i}+\beta_{i}\right) d_{i}^{*}-\beta_{i}\left(\gamma_{i}+\alpha_{i}\right) d_{i+1}^{*} \\
& =4 \alpha_{i} \beta_{i} \triangle_{i}^{*}+\gamma_{i}^{2} \triangle_{i}^{*}+\gamma_{i} \alpha_{i} \triangle_{i}^{*}+\gamma_{i} \beta_{i} \triangle_{i}^{*}-\gamma_{i} \alpha_{i} d_{i}^{*}-\gamma_{i} \beta_{i} d_{i+1}^{*}-\alpha_{i} \beta_{i} d_{i}^{*}-\alpha_{i} \beta_{i} d_{i+1}^{*} .
\end{aligned}
$$

Substituting $\gamma_{i}$ (see equation (32)) in the above expression, we get

$$
\begin{aligned}
A_{3, i}= & 4 \alpha_{i} \beta_{i} \triangle_{i}^{*}+\frac{\left(\alpha_{i} d_{i}^{*}+\beta_{i} d_{i+1}^{*}\right)^{2}}{\triangle_{i}^{*}}+\alpha_{i}\left(\alpha_{i} d_{i}^{*}+\beta_{i} d_{i+1}^{*}\right)+\beta_{i}\left(\alpha_{i} d_{i}^{*}+\beta_{i} d_{i+1}^{*}\right) \\
& -\frac{\alpha_{i} d_{i}^{*}\left(\alpha_{i} d_{i}^{*}+\beta_{i} d_{i+1}^{*}\right)}{\triangle_{i}^{*}}-\frac{\beta_{i} d_{i+1}^{*}\left(\alpha_{i} d_{i}^{*}+\beta_{i} d_{i+1}^{*}\right)}{\triangle_{i}^{*}}-\alpha_{i} \beta_{i} d_{i}^{*}-\alpha_{i} \beta_{i} d_{i+1}^{*} \\
= & 4 \alpha_{i} \beta_{i}\left(\triangle_{i}-\xi_{i} \frac{f_{n}-f_{1}}{x_{n}-x_{1}}\right)+\alpha_{i}^{2}\left(d_{i}-\xi_{i} d_{1}\right)+\beta_{i}^{2}\left(d_{i+1}-\xi_{i} d_{n}\right) .
\end{aligned}
$$

From the final expression of $A_{3, i}$, it is easy to verify that equations (32) and (34)-(36) are sufficient for $A_{3, i} \geq 0$. Hence we have proved that the fixed point $\psi$ of the rational cubic IFS (11) is monotonically increasing over $\left[x_{1}, x_{n}\right]$, if the scaling factors and shape parameters are chosen according to equation (31) and equation (32), respectively. In the case of $\triangle_{i}=0$, the fixed point of the rational cubic IFS (11) is a constant throughout that subinterval with the value $f_{i}$, and $\xi_{i}=0$.

\section{Case II: Monotonically decreasing data}

Suppose $\left\{\left(x_{i}, f_{i}\right), i=1,2, \ldots, n\right\}$ is a given monotonically decreasing data set. It is easy to see that the sufficient conditions for monotonicity of equation (10) on $\left[x_{i}, x_{i+1}\right]$ are $A_{j, i} \leq 0$, $j=1,2, \ldots, 5$. As explained in Case I, it is easy to verify that selections of the scaling factors and shape parameters according to equation (31) and (32), respectively, are sufficient for $A_{j, i} \leq 0, j=1,2, \ldots, 5$.

Therefore from the arguments in Case I and Case II, we conclude that if the scaling factors and shape parameters are chosen according to (31) and (32), respectively, then the fixed point $\psi$ of the rational cubic IFS (11) is monotone for given monotonic data.

Remark 3 Convergence results in Corollary 1 are valid for the shape-preserving rational cubic FIFs.

\subsection{Examples and discussion}

We construct the $\mathcal{C}^{1}$-rational cubic fractal interpolation functions (RCFIFs) for the standard increasing Akima data $[26]\{(0,10),(2,10),(3,10),(5,10),(6,10),(8,10),(9,10.5),(11,15)$, 


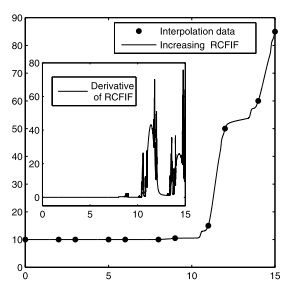

(a) A standard rational cubic FIF.

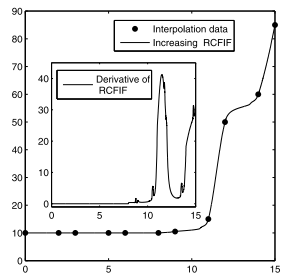

(d) Effects of $\xi_{7}, \xi_{8}, \xi_{9}$ and $\xi_{10}$ in Figure 3(a).

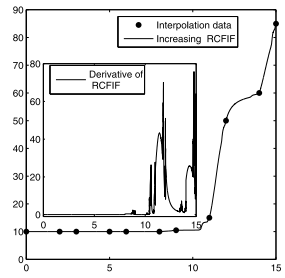

(g) Effects of $\alpha_{9}$ in Figure 3(c).

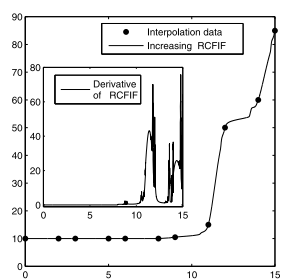

(b) Effects of $\xi_{7}$ in Figure 3(a).

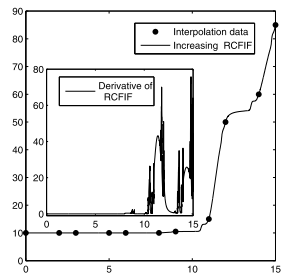

(e) Effects of $\alpha_{9}$ and $\beta_{9}$ in Figure 3(a).

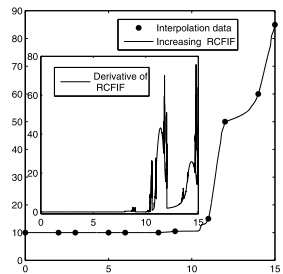

(h) Effects of $\beta_{9}$ in Figure 3(c).

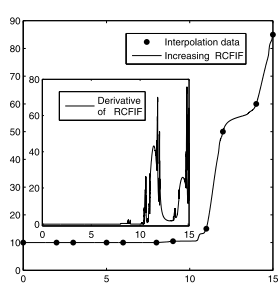

(c) Effects of $\xi_{9}$ in Figure 3(a).

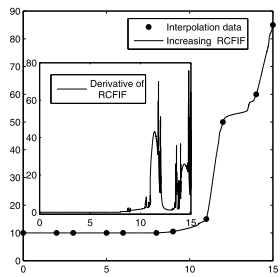

(f) Effects of $\alpha_{7}$ and $\beta_{7}$ in Figure 3(b).

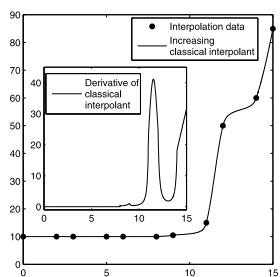

(i) Classical rational cubic interpolant of Figure 3(g).

Figure 3 Monotonicity preserving rational cubic FIFs and their derivatives.

$(12,50),(14,60),(15,85)\}$. The $\mathcal{C}^{1}$-rational cubic FIFs are generated iteratively (Figures 3(a)(i)) as the fixed points of rational cubic IFS (11). Since $\Delta_{i}=0$ for $i=1,2, \ldots, 5, \xi_{i}=0$ for $i=1,2, \ldots, 5$, and there is no need to choose $\alpha_{i}$ and $\beta_{i}$, consequently there is no need to calculate $\gamma_{i}$ for $i=1,2, \ldots, 5$ using equation (32). The derivatives values $d_{i}(i=1,7, \ldots, 11)$ are approximated by the arithmetic mean method [24] as $d_{1}=0, d_{2}=0, d_{3}=0, d_{4}=0$, $d_{5}=0, d_{6}=0.3333, d_{7}=1.0833, d_{8}=24.0833, d_{9}=25, d_{10}=18.333$, and $d_{11}=31.6667$. Let $\kappa=0.9999$ in equation (31). The scaling factors are restricted as $\xi_{6} \in[0,0.0342]$, $\xi_{7} \in[0,0.45], \xi_{8} \in[0,0.7895], \xi_{9} \in[0,0.5789], \xi_{10} \in[0,0.9999)$ to capture the monotonicity of the Akima data. A standard rational cubic FIF $\Phi$ in Figure 3(a) is generated with a suitable choice of the scaling factors (see Table 3). By comparing Figure 3(b) with Figure 3(a), we observe that the graph of the rational cubic FIF in the subinterval $\left[x_{7}, x_{8}\right]$ converges to a convex shape as $\xi_{7} \rightarrow 0^{+}$, and the changes in other intervals are negligible. By analyzing the shapes of Figure 3(a) and Figure 3(c), we observe that visually pleasing effects are produced in the ninth subinterval. By analyzing Figure 3(d) with respect to Figure 3(a), we have found excellent variations in $\left[x_{7}, x_{10}\right]$. The individual effects of $\xi_{7}$ and $\xi_{9}$, respectively, from Figure 3(b) and Figure 3(c) are reflected in Figure 3(d), and thereby we conclude that all the non-zero scaling factors are very much local in nature for this Akima data. Next to visualize the effects of change in the shape parameters, we construct the monotonically increasing rational cubic FIFs in Figures 3(e)-(h). By comparing the shapes 
Table 3 Rational IFS parameters for monotonic fractal interpolants

\begin{tabular}{|c|c|c|}
\hline Figure & Scaling factors & Shape parameters \\
\hline $3(\mathrm{a})$ & $\begin{array}{l}\xi_{6}=0.034, \xi_{7}=0.44 \\
\xi_{8}=0.789, \xi_{9}=0.578, \xi_{10}=0.999\end{array}$ & $\begin{array}{l}\alpha_{i}=\beta_{i}=1, i=6(1) 10, \gamma_{6}=4.2927 \\
\gamma_{7}=503.3320, \gamma_{8}=1.5805 \\
\gamma_{9}=20.5370, \gamma_{10}=2.4994\end{array}$ \\
\hline $3(b)$ & $\begin{array}{l}\xi_{6}=0.034, \xi_{7}=\mathbf{0 . 1} \\
\xi_{8}=0.789, \xi_{9}=0.578, \xi_{10}=0.999\end{array}$ & $\begin{array}{l}\alpha_{i}=\beta_{i}=1, i=6(1) 10, \gamma_{6}=4.2927 \\
\gamma_{7}=\mathbf{1 4 . 3 8 0 8}, \gamma_{8}=1.5805 \\
\gamma_{9}=20.5370, \gamma_{10}=2.4994\end{array}$ \\
\hline $3(c)$ & $\begin{array}{l}\xi_{6}=0.034, \xi_{7}=0.44 \\
\xi_{8}=0.789, \xi_{9}=\mathbf{0 . 1}, \xi_{10}=0.999\end{array}$ & $\begin{array}{l}\alpha_{i}=\beta_{i}=1, i=6(1) 10, \gamma_{6}=4.2927 \\
\gamma_{7}=503.3320, \gamma_{8}=1.5805 \\
\gamma_{9}=\mathbf{9 . 6 2 9 6}, \gamma_{10}=2.4994\end{array}$ \\
\hline $3(d)$ & $\begin{array}{l}\xi_{6}=0.034, \xi_{7}=\mathbf{0 . 1} \\
\xi_{8}=\mathbf{0 . 1}, \xi_{9}=\mathbf{0 . 1}, \xi_{10}=\mathbf{0 . 1}\end{array}$ & $\begin{array}{l}\alpha_{i}=\beta_{i}=1, i=6(1) 10, \gamma_{6}=4.2927 \\
\gamma_{7}=\mathbf{1 4 . 3 8 0 8}, \gamma_{8}=\mathbf{1 . 4 2 2 7} \\
\gamma_{9}=\mathbf{2 0 . 5 3 7}, \gamma_{10}=\mathbf{2 . 0 4 0 8}\end{array}$ \\
\hline $3(\mathrm{e})$ & $\begin{array}{l}\xi_{6}=0.034, \xi_{7}=0.44 \\
\xi_{8}=0.789, \xi_{9}=0.578, \xi_{10}=0.999\end{array}$ & $\begin{array}{l}\alpha_{i}=\beta_{i}=1, i \in\{6,7,8,10\}, \alpha_{9}=\mathbf{1 0}^{\mathbf{4}}, \beta_{9}=\mathbf{1 0} \\
\alpha_{10}=1, \beta_{10}=1, \gamma_{6}=4.2927, \gamma_{7}=503.3320 \\
\gamma_{8}=1.5805, \gamma_{9}=\mathbf{1 . 1 8 5 7}, \gamma_{10}=2.4994\end{array}$ \\
\hline $3(f)$ & $\begin{array}{l}\xi_{6}=0.034, \xi_{7}=\mathbf{0 . 1} \\
\xi_{8}=0.789, \xi_{9}=0.578, \xi_{10}=0.999\end{array}$ & $\begin{array}{l}\alpha_{i}=\beta_{i}=1, i \in\{6,8,9,10\} \\
\alpha_{7}=\mathbf{1 0}^{\mathbf{4}}, \beta_{7}=\mathbf{1 0}, \gamma_{6}=4.2927, \gamma_{7}=\mathbf{6 . 3 2 7 6} \\
\gamma_{8}=1.5805, \gamma_{9}=20.5370, \gamma_{10}=2.4994\end{array}$ \\
\hline $3(\mathrm{~g})$ & $\begin{array}{l}\xi_{6}=0.034, \xi_{7}=0.44 \\
\xi_{8}=0.789, \xi_{9}=\mathbf{0 . 1}, \xi_{10}=0.999\end{array}$ & $\begin{array}{l}\alpha_{i}=\beta_{i}=1, i \in\{6,7,8,10\} \\
\alpha_{9}=\mathbf{1 0}^{\mathbf{4}}, \beta_{9}=1, \gamma_{6}=4.2927, \gamma_{7}=503.3320 \\
\gamma_{8}=1.5805, \gamma_{9}=\mathbf{5 . 5 5 6}, \gamma_{10}=2.4994\end{array}$ \\
\hline $3(h)$ & $\begin{array}{l}\xi_{6}=0.034, \xi_{7}=0.44 \\
\xi_{8}=0.789, \xi_{9}=\mathbf{0 . 1}, \xi_{10}=0.999\end{array}$ & $\begin{array}{l}\alpha_{i}=\beta_{i}=1, i \in\{6,7,8,10\} \\
\alpha_{9}=1, \beta_{9}=\mathbf{1 0}^{\mathbf{4}}, \gamma_{6}=4.2927, \gamma_{7}=503.3320 \\
\gamma_{8}=1.5805, \gamma_{9}=\mathbf{4 . 0 7 9 6}, \gamma_{10}=2.4994\end{array}$ \\
\hline $3(i)$ & $\xi_{i}=\mathbf{0}, i=6(1) 10$ & $\begin{array}{l}\alpha_{i}=\beta_{i}=1, i \in\{6,7,8,10\}, \\
\alpha_{9}=10^{4}, \beta_{9}=1, \gamma_{6}=\mathbf{0 . 0 0 0 3}, \gamma_{7}=\mathbf{0 . 0 0 1 1}, \\
\gamma_{8}=\mathbf{0 . 0 0 0 1}, \gamma_{9}=\mathbf{5 . 0 0 0 4}, \gamma_{10}=\mathbf{0 . 0 0 0 2}\end{array}$ \\
\hline
\end{tabular}

of Figure 3(e) and Figure 3(a), we notice perceptible variations in the ninth subinterval, and variations in other subintervals are negligible. Again analyzing Figure 3(a), Figure 3(c), and Figure 3(e), we observe that to get appropriate deviations in the rational cubic FIF in the ninth subinterval, one has to vary the scaling parameter $\xi_{9}$, the shape parameters $\alpha_{9}$ and/or $\beta_{9}$ suitably.

Next a monotonic rational FIF in Figure 3(f) is constructed as per the data in Table 3 and the variations in $\left[x_{7}, x_{8}\right]$ of Figure 3(f) with respect to Figure 3(a) are more evident than those of Figure 3(f) with respect to Figure 3(b). So, we can say that the scaling factor $\xi_{7}$ is dominant over the shape parameters $\alpha_{7}$ and $\beta_{7}$ in this case at $\left[x_{7}, x_{8}\right]$. Hence, for major changes at $\left[x_{7}, x_{8}\right]$, one has to modify $\xi_{7}$, and for minor changes (or fine tuning), one has to alter $\alpha_{7}$ and/or $\beta_{7}$. This observation is useful for aesthetic requirements in various engineering design problems. By analyzing Figures 3(g)-(h) with respect to Figure 3(c), we have noticed that the graphs of rational cubic FIFs in the ninth subinterval in Figures 3(g)(h) are concave and convex, respectively. Finally, we construct the classical rational cubic interpolant in Figure 3(i) with respect to the shape parameters of Figure 3(g), and $\xi_{i}=0$ for all $i$. Since the shape parameters $\alpha_{9}$ and $\beta_{9}$ are the same in Figure 3(g) and Figure 3(i), there is some visual similarity between these two curves in $\left[x_{7}, x_{8}\right]$, whereas the same effects are missing in Figure 3(h) due to a variation in $\beta_{9}$. Also, one gets a classical rational cubic interpolant which is similar to Figure 3(h) in our fractal scheme, whenever $\xi_{i}=0$ for all $i$, and the shape parameters are chosen according to Figure 3(h). The presence of scaling factors in a monotonic rational FIF with shape parameters gives an additional advantage in the choice of interpolant over the classical interpolants with shape parameters. 
Table 4 Uniform errors between $\Phi$ and rational fractal interpolants, and their derivatives

\begin{tabular}{llll}
\hline $\begin{array}{l}\text { Monotonic } \\
\text { RCFIF }\end{array}$ & $\begin{array}{l}\text { Uniform distance with } \\
\text { monotonic RCFIF in Figure 3(a) }\end{array}$ & $\begin{array}{l}\text { Derivative of } \\
\text { monotonic RCFIF }\end{array}$ & $\begin{array}{l}\text { Uniform distance with } \\
\text { derivative in Figure 3(a) }\end{array}$ \\
\hline Figure 3(b) & 0.7871 & Figure 3(b) & 18.736 \\
Figure 3(c) & 2.3678 & Figure 3(c) & 27.0239 \\
Figure 3(d) & 2.3783 & Figure 3(d) & 44.6254 \\
Figure 3(e) & 0.8223 & Figure 3(e) & 3.6775 \\
Figure 3(f) & 2.0038 & Figure 3(f) & 18.6884 \\
Figure 3(g) & 4.6438 & Figure 3(g) & 29.531 \\
Figure 3(h) & 1.5456 & Figure 3(h) & 23.4984 \\
\hline
\end{tabular}

Our construction gives an extra freedom for aesthetic modifications in local shape over the classical rational cubic interpolants to an user. For a qualitative study of the derivatives of monotonic fractal interpolants, the readers are invited to check the effects of rational IFS parameters in Figures 3(a)-(g). The uniform errors between monotonic fractal interpolants and their derivatives are given in Table 4 to show the importance of our rational cubic IFS (11).

From the examples in Sections 4-5, it is observed that proper interactive adjustments of the scaling factors and shape parameters give us a wide variety of positivity and/or monotonicity preserving fixed points of our rational cubic IFS (11) that can be used in various scientific and engineering problems for aesthetic modifications. In order to get an optimal choice of the fixed point of our rational cubic IFS (11), one can employ a genetic algorithm interactively until the desired accuracy is obtained with the original function.

\section{Conclusion}

A new type of rational cubic IFS with 3-shape parameters is introduced in this work such that its fixed point can be used for shaped data. The developed FIF in this paper includes the corresponding classical rational cubic interpolant as a special case. An upper bound of uniform error between the rational cubic FIF $\psi$ and an original function $\Phi$ in $\mathcal{C}^{4}\left[x_{1}, x_{n}\right]$ is estimated, and consequently we have found that $\psi$ converges uniformly to $\Phi$ as $h \rightarrow 0$. When the accurate derivatives of $O\left(h_{i}^{p}\right), i=1,2, \ldots, n$ are available, and the scaling factors are chosen as $\left|\xi_{i}\right|<\kappa a_{i}^{p-1}, i=1,2, \ldots, n-1$, it is possible to get $O\left(h^{p}\right)(p=2,3,4)$ convergence for the rational cubic FIF. Automatic data dependent restrictions are derived on the scaling factors and shape parameters of rational cubic IFS so that its fixed point preserves the positivity or monotonicity features of a given set of data. The effects of a change in the scaling factors and shape parameters on the local control of the shape of rational cubic FIF are demonstrated through various examples. Our rational cubic FIFs are more flexible and more suitable for shape related problems in computer graphics, CAD/CAM, CAGD, medical imaging, finance, and engineering applications, and apply equally well to data with or without derivatives. In particular, the proposed method will be an ideal tool in shape-preserving interpolation problems where the data set originates from a positive and/or monotonic function $\Phi \in \mathcal{C}^{1}$, but its derivative $\Phi^{\prime}$ is a continuous and nowhere differentiable function.

Competing interests

The authors declare that they have no competing interests. 


\section{Author details}

${ }^{1}$ Department of Mathematics, Indian Institute of Technology Madras, Chennai, 600036, India. ${ }^{2}$ Department of Mathematics, Texas A\&M University - Kingsville, 700 University Blvd, Kingsville, TX 78363-8202, USA. ${ }^{3}$ Department of Mathematics, Faculty of Science, King Abdulaziz University, Jeddah, 21589, Saudi Arabia.

\section{Acknowledgements}

AKBC is thankful to the Department of Science and Technology, Govt. of India for the SERC DST Project No. SR/S4/MS $694 / 10$. The authors are grateful to the anonymous referees for the valuable comments and suggestions, which improved the presentation of the paper.

\section{Received: 2 July 2013 Accepted: 16 December 2013 Published: 27 Jan 2014}

\section{References}

1. Mandelbrot, BB: Fractals: Form, Chance and Dimension. Freeman, San Francisco (1977)

2. Feder, J: Fractals. Plenum Press, New York (1988)

3. West, BJ: Fractals in Physiology and Chaos in Medicine. World Scientific, Singapore (1990)

4. Yang, YJ, Baleanu, D, Yang, XJ: Analysis of fractal wave equations by local fractional Fourier series method. Adv. Math. Phys. 2013, Article ID 632309 (2013)

5. Hutchinson, JE: Fractals and self similarity. Indiana Univ. Math. J. 30, 713-747 (1981)

6. Barnsley, MF: Fractals Everywhere. Academic Press, Orlando, Florida (1988)

7. Barnsley, MF: Fractal functions and interpolation. Constr. Approx. 2, 303-329 (1986)

8. Barnsley, MF, Harrington, AN: The calculus of fractal interpolation functions. J. Approx. Theory 57, 14-34 (1989)

9. Chand, AKB, Kapoor, GP: Generalized cubic spline fractal interpolation functions. SIAM J. Numer. Anal. 44(2), 655-676 (2006)

10. Chand, AKB, Navascués, MA: Generalized Hermite fractal interpolation. Rev. Acad. Cienc. Exactas, Fís.-Quím. Nat. Zaragoza 64, 107-120 (2009)

11. Chand, AKB, Navascués, MA: Natural bicubic spline fractal interpolation. Nonlinear Anal. 69, 3679-3691 (2008)

12. Chand, AKB, Viswanathan, P: Cubic Hermite and cubic spline fractal interpolation functions. AIP Conf. Proc. 1479, $1467-1470(2012)$

13. Chand, AKB, Viswanathan, P: A constructive approach to cubic Hermite fractal interpolation function and its constrained aspects. BIT Numer. Math. 53(4), 841-865 (2013)

14. Schmidt, JW, Heß, W: Positivity of cubic polynomials on intervals and positive spline interpolation. BIT Numer. Math. 28, 340-352 (1988)

15. Fritsch, FN, Carlson, RE: Monotone piecewise cubic interpolation. SIAM J. Numer. Anal. 17, 238-246 (1980)

16. Schumaker, LL: On shape preserving quadratic spline interpolation. SIAM J. Numer. Anal. 20, 854-864 (1983)

17. Brodlie, KW, Butt, S: Preserving convexity using piecewise cubic interpolation. Comput. Graph. 15, 15-23 (1991)

18. Späth, H: Spline Algorithms for Curves and Surfaces. Utilias Mathematica Pub. Inc., Winnipeg (1974)

19. Delbourgo, R, Gregory, JA: Shape preserving piecewise rational interpolation. SIAM J. Sci. Stat. Comput. 6, 967-976 (1985)

20. Sarfraz, M, AL-Muhammed, M, Ashraf, F: Preserving monotonic shape of the data using piecewise rational cubic functions. Comput. Graph. 21, 5-14 (1997)

21. Sarfraz, M, Hussain, MZ, Hussain, M: Shape-preserving curve interpolation. Int. J. Comput. Math. 89, 35-53 (2012)

22. Kwakernaak, H, Sivan, R: Linear Optimal Control Systems. Wiley-Interscience, New York (1972)

23. Gregory, JA, Delbourgo, R: Piecewise rational quadratic interpolation to monotonic data. IMA J. Numer. Anal. 2 , 123-130 (1982)

24. Gregory, JA, Delbourgo, R: Determination of derivative parameters for a monotonic rational quadratic interpolant. IMA J. Numer. Anal. 5, 397-406 (1985)

25. Chand, AKB, Vijender, N: Monotonicity preserving rational quadratic fractal interpolation functions. Adv. Numer. Anal. 2014, Article ID 504825 (2014)

26. Akima, $\mathrm{H}$ : A new method of interpolation and smooth curve fitting based on local procedures. J. Assoc. Comput. Mach. 17, 589-602 (1970)

10.1186/1687-1847-2014-30

Cite this article as: Chand et al.: Rational iterated function system for positive/monotonic shape preservation.

Advances in Difference Equations 2014, 2014:30 